\title{
INNOVACIÓN EDUCATIVA UNIVERSITARIA Y METODOLOGÍAS ACTIVAS PARA EL APRENDIZAJE DE LAS COMPETENCIAS ESPECÍFICAS DEL GRADO
}

\author{
UNIVERSITY EDUCATIONAL INNOVATION AND ACTIVE \\ METHODOLOGIES FOR THE LEARNING OF THE SPECIFIC \\ COMPETENCES OF THE DEGREE
}

\section{Rocio Fernández Piqueras ${ }^{a}$, Empar Guerrero Valverde ${ }^{a^{*}}$, Sara Cebrián Cifuentes ${ }^{a}$ y Concepción Ros $\operatorname{Ros}^{a}$}

Fechas de recepción y aceptación: 30 de septiembre de 2020 y 8 de noviembre de 2020

DOI: https://doi.org/10.46583/edetania_2020.58.723

Resumen: Este artículo presenta los resultados de la percepción del aprendizaje de una experiencia educativa innovadora llevada a cabo en la Universidad Católica de Valencia en el marco de la III Convocatoria de proyectos de innovación docente y premios a la Innovación 2019/20 que involucra a tres asignaturas de grado de dos facultades distintas. Uno de los mayores retos de los docentes universitarios es lograr que sus estudiantes desarrollen las competencias específicas que les permitan obtener un aprendizaje con el que poder adaptarse a este mundo cambiante, siendo al mismo tiempo productivos, innovadores y creativos. Para ello las metodologías activas como la gamificación se convierten en buenos aliados que involucran al estudiante en su proceso de aprendizaje logrando el desarrollo de dichas competencias. El diseño básico de la investigación es cuasiexperimental postest, basado en la autopercepción de los estudiantes sobre su aprendizaje en las competencias específicas de las asignaturas después de la realización del proyecto. Los análisis estadísticos descriptivos se han realizado con el programa SPSS v23, obteniendo unos resultados que evidencian el aprendizaje positivo del estudiante en función de las variables personales y contextuales.

Palabras clave: proyectos de innovación docente, aprendizaje por competencias, metodologías activas.

\footnotetext{
${ }^{a}$ Facultad de Magisterio y Ciencias de la Educación. Universidad Católica de Valencia San Vicente Mártir

${ }^{*}$ Correspondencia: Universidad Católica de Valencia San Vicente Mártir. Facultad de Magisterio y Ciencias de la Educación. Calle Sagrado Corazón, 5. 46110 (Godella), Valencia. España.

E-mail: empar.guerrero@ucv.es
} 
Abstract: This article presents the results of the perception of learning of an innovative educational experience carried out at the Catholic University of Valencia within the framework of the III Call for teaching innovation projects and 2019/20 Innovation Awards involving three undergraduate subjects of two different faculties. One of the greatest challenges for university teachers is to ensure that their students develop the specific skills that allow them to obtain learning with which to adapt to this changing world, while being productive, innovative and creative at the same time. For this, active methodologies such as gamification become good allies that involve the student in their learning process, achieving the development of said skills. The basic design of the research is quasi-experimental post-test, based on the students' self-perception of their learning in the specific competencies of the subjects after the completion of the project. Descriptive statistical analyses were carried out with the SPSS v23 program, obtaining results that show positive student learning based on personal and contextual variables.

Keywords: teaching innovation projects, learning by competencies, active methodologies.

\section{INTRODUCCIÓN}

Con la adaptación al plan Bolonia se ha visto la necesidad de introducir cambios metodológicos para adecuarse a las nuevas realidades actuales. El diseño curricular basado en competencias, la incorporación de un alumnado nativo digital y la aparición de las tecnologías de la información y la comunicación en el ámbito educativo son tres aspectos que han conducido a la introducción de nuevas formas de enseñar en la Universidad.

La sociedad reclama un nuevo modelo educativo que incorpore metodologías docentes que fomenten un aprendizaje significativo, experiencial y cooperativo, en el que el estudiante sea parte activa del proceso. Todo ello ha puesto en cuestión el modelo tradicional que transmite los conocimientos de forma estandarizada y fragmentada (Morín, 2001), sin tener en cuenta los significados personales y la parte activa del aprendizaje en el estudiante; se centra más en el que enseña que en el que aprende. Sin embargo este modelo no funciona, es necesario captar la atención del estudiante mediante el ofrecimiento de propuestas de trabajo diferentes y la incorporación de elementos novedosos que causen sorpresa.

Las corrientes pedagógicas centradas en los procesos de aprendizaje, junto con los conocimientos que nos aporta la neurociencia, están contribuyendo a entender que las aulas deben convertirse en un espacio dinámico en el que 
se intercambien conocimientos, experiencias o reflexiones, y en el que el estudiante se convierta en verdadero protagonista del proceso de aprendizaje (Bueno, 2019). Los docentes deben ser generadores de desafíos que hagan cuestionarse al alumnado el significado de lo que aprende, que este pueda otorgarle sentido a lo aprendido y sea capaz de transferir estos conocimientos a contextos diferentes.

Para que esto se dé, la Universidad deberá innovar en sus procesos de enseñanza-aprendizaje y proporcionar medios diferentes que ayuden al estudiante a generar nuevas ideas de forma creativa, adquirir unas competencias adaptadas a las necesidades del mercado laboral y aprender a responder de forma adecuada a las exigencias del entorno.

Otro aspecto que interviene en el proceso de aprendizaje y que deberá tenerse en cuenta es la importancia que las emociones tienen en dicho proceso. Se sostiene que las emociones son una parte esencial en el aprendizaje ya que activan y estimulan el cerebro (Bueno, 2019). Además, las emociones positivas contribuyen a consolidar la memoria a largo plazo y al proceso lógico del pensamiento. Por ello, resulta indispensable que se introduzcan con frecuencia elementos novedosos y atractivos en el aula que llamen la atención y despierten la curiosidad en el estudiante. Así como utilizar estrategias prácticas que fomenten la creatividad y que les permitan ser los protagonistas en el proceso de aprendizaje sin ser meros elementos pasivos en el aula, articulando los contenidos curriculares y las competencias profesionales específicas que deben adquirir.

\section{LA INNOVACIÓN EN EL AULA UNIVERSITARIA}

Innovar implica reflexionar, diseñar, aplicar e implantar elementos de proceso o de producto que suponen siempre pasar de una situación determinada a otra mejor. Es, en consecuencia, promover un nuevo proceso, un nuevo producto, servicio o nuevo conocimiento (Corbo, Reinholz, Dancy, Deetz y Finkelstein, 2016). Diferentes autores la definen como "una idea, práctica o proyecto que es percibida como nueva por un individuo u otra unidad de adopción" (Rogers, 2003: 11); también como el proceso de elaboración de nuevos productos que pueden adoptarse o rediseñarse para su uso y transformación 
(Rikkerink, Verbeeten, Simons y Ritzen, 2016), e incluso de forma abierta (Ramírez-Montoya, 2018). Los nuevos procesos, productos, servicios y conocimientos son los motores del cambio en el ámbito de la educación, en el que la innovación suele contribuir a abordar los problemas y situaciones derivados de las prácticas docentes y la prestación de servicios. La innovación educativa significa realizar cambios en el aprendizaje y en la formación para mejorar los resultados del aprendizaje (Fidalgo, Sein, García, Balbín, 2019).

La innovación va acompañada de la selección, organización y utilización creativa de recursos humanos y materiales de maneras nuevas y propias que den como resultado la conquista de un nivel más alto con respecto a las metas y objetivos previamente marcados. Por tanto, la innovación, para serlo, debe producir un cambio intencional y deliberado. Todo proyecto de innovación surge de la detección de una carencia, o de un deseo de mejora, a partir de la cual se impone el análisis de la situación que se quiere cambiar y optimizar. Asimismo, cuenta con tres soportes básicos: el productivo, el técnico y la creatividad.

La innovación educativa se genera a partir del análisis de las necesidades del alumnado, la implementación (experimentación) de nuevos modelos de enseñanza-aprendizaje y la adaptación de los contenidos a las nuevas realidades sociales. En este escenario, las universidades se convierten en espacios idóneos para producir nuevos conocimientos y contribuir al crecimiento de las sociedades porque desempeñan un papel fundamental en tres ámbitos: la investigación y explotación de sus resultados; la educación y la formación; y el desarrollo regional y local al que pueden contribuir de manera significativa. La educación superior debe adaptarse a las transformaciones sociales que se producen de forma continua y responder a los retos de la globalización con eficacia. Adaptando, además, las nuevas tendencias o modelos digitales que aportan nuevas posibilidades al mundo educativo convirtiéndose en un instrumento eficaz para transformar el modelo pedagógico de nuestras instituciones educativas. La revisión previa de la literatura en temas referentes a innovación educativa indica que los temas emergentes en dicha innovación hacen referencia a la educación digital, las tecnologías adaptativas, tecnologías abiertas, inteligentes y disruptivas (González, Ramírez, García y Martí, 2019; Ramírez-Montaya y Lugo-Ocando; 2020). 
La Universidad debe abrirse más a la sociedad, conectarse a la realidad, reforzando los vínculos entre enseñanza superior, el mundo del trabajo y otros sectores sociales. La conexión entre la teoría y la práctica debe estar presente. Ya no basta con que el alumno acumule conocimientos, sino que se debe promover la combinación de conocimientos teóricos y prácticos, superando la separación existente entre ambas. Y todo ello supone un reto importante para los docentes universitarios ya que deben formar a ciudadanos activos, centrando el aprendizaje en la adquisición y desarrollo de competencias por parte del alumnado que se adecuen al mercado laboral y a una sociedad cambiante.

La innovación es un proceso complejo, que se alimenta tanto del conocimiento tácito como del conocimiento observable y especializado, influido por múltiples factores, algunos internos e individuales (motivación, proactividad, etc.) y otros externos, que proceden del entorno (Robinson, 2015). En este momento, la innovación educativa es un hecho cada vez más presente en las aulas universitarias, produciéndose cambios en la forma de dar la clase, así como modificando o adaptando el contenido a los nuevos avances sociales, políticos, económicos o culturales, y por supuesto ajustando todo ello a lo que necesita el alumno del siglo Xxi (Barnet, 2001). Estos alumnos deben ser preparados para un futuro profesional y capacitarles para su desarrollo personal.

Para dar respuesta a todo ello, se hace necesario introducir métodos innovadores de enseñanza-aprendizaje que den respuesta a estas nuevas realidades educativas. En este sentido, se han venido implementando metodologías activas en las aulas, entendiéndolas como aquellos métodos, estrategias y técnicas que utiliza el docente para convertir el proceso de enseñanza-aprendizaje en una propuesta educativa integrada por actividades que fomentan la participación del estudiante y que tienen como finalidad el aprendizaje de nuevos conocimientos y la adquisición de competencias, así como el desarrollo de capacidades.

\section{LAS METODOLOGÍAS ACTIVAS Y EL APRENDIZAJE POR COMPETENCIAS}

Las competencias son entendidas como "el buen desempeño en contextos diversos y auténticos basado en la integración y activación de conocimientos, normas, técnicas, procedimientos, habilidades y destrezas, actitudes y 
valores" (Villa y Poblete, 2010, p. 23). Este concepto se introduce en nuestras universidades en la primera década del siglo XXI a consecuencia del proceso de Bolonia. "El EEES plantea un contexto diferente que exige que la universidad proporcione una formación más amplia, dotando a los estudiantes de muchas más cualidades, las cuales les van a ser necesarias cuando se incorporen a la sociedad al finalizar sus estudios" (Leví y Ramos, 2013, p. 626).

Para aprender y desarrollar una competencia se deben combinar conocimientos de carácter teórico, procedimental y actitudinal, y para ello se han diseñado competencias instrumentales (suponen una combinación de habilidades manuales y capacidades cognitivas que posibilitan la competencia profesional); competencias interpersonales (se refieren a la capacidad, habilidad o destreza en expresar los propios sentimientos y emociones del modo más adecuado y aceptando los sentimientos de los demás); y competencias sistémicas (estas requieren haber adquirido previamente las anteriores ya que suponen destrezas y habilidades relacionadas con la totalidad de un sistema) (Villa y Poblete, 2010).

Además, en el Real Decreto 1393/2007 de Ordenación de las Enseñanzas Universitarias se concreta que las competencias genéricas son aquellas que resulten comunes a todas las disciplinas (liderazgo, trabajo en equipo, creatividad, etc.), y las competencias específicas son las que están vinculadas con el conocimiento concreto de una disciplina.

Para materializar planes de estudios universitarios basados en este modelo competencial, dichos planes adquieren una concepción más interdisciplinar e incorporan un nuevo modelo pedagógico pasando de una orientación centrada en la enseñanza a una orientación basada en el aprendizaje, cambiando el papel del profesorado y del alumnado. Esto, además, va acompañado de la necesaria implementación de metodologías didácticas más activas, participativas y dinámicas, que favorecen la conexión entre la teoría y la práctica y convierten al estudiante en protagonista del proceso de enseñanza-aprendizaje (De Miguel, 2006). "Existe un amplio consenso en considerar que las estrategias metodológicas más activas potencian la implicación del alumno en su propio aprendizaje y son muy importantes porque refuerzan su responsabilidad, autoestima, interés y motivación" (Romero, 2011, p. 368).

Estas propuestas metodológicas deben integrar una serie de elementos como son: conocimientos, técnicas, actitudes y valores que "una persona 
pone en juego en una situación concreta demostrando que es capaz de resolverla" (Villa y Poblete, 2011, p. 148), y deben partir de principios tales como:

- Adecuarse al desarrollo del alumnado y de sus aprendizajes previos.

- Favorecer aprendizajes significativos.

- Potenciar en el alumnado la capacidad de razonar y de ser críticos.

- Facilitar el trabajo cooperativo.

- Dotar al alumnado de herramientas para resolver conflictos.

- Trabajar para que los estudiantes adquieren la capacidad de aprender a aprender.

Existen actualmente algunas metodologías basadas en estos principios y que en las últimas décadas se han ido consolidando en los diferentes niveles educativos. Entre estas metodologías encontramos: el aprendizaje basado en proyectos, flipped classroom, talleres didácticos o la gamificación, siendo esta última en la que se ha basado la propuesta de enseñanza-aprendizaje, para la adquisición de competencias de alumnado universitario de los grados de Educación Social y Ciencias de la Actividad Física y del Deporte.

La gamificación emerge como una herramienta de transformación educativa (Corchuelo, 2018). Es un proceso en el que se aplican mecánicas de juegos, en contextos que no son propiamente lúdicos, y que favorece la motivación del alumnado para la consecución de ciertos objetivos. El punto clave es que el juego puede integrarse en actividades que no son en sí mismas un juego (Werbach y Hunter, 2012). La propuesta educativa gamificada debe organizarse, estructurarse y sistematizarse. Está compuesta por dinámicas centradas en retos, recompensas, logros, etc., lo cual ayuda al docente a transformar clases formativas, o tareas aburridas, en atractivos momentos educativos de aprendizaje significativo, en donde además de mejorar sus resultados académicos llevan a cabo una participación activa de la clase (Oliva, 2016).

\section{DisEÑO DE LA INNOVACIÓN EDUCATIVA REALIZADA}

El diseño básico de la investigación es un diseño cuasiexperimental postest, basado en un diseño de encuesta propia sobre la autopercepción del aprendizaje, de tipo transversal. 


\subsection{Contexto y participantes}

La experiencia innovadora se ha llevado a cabo con 86 estudiantes de dos grados de la Universidad Católica San Vicente Mártir, durante el curso 20192020. Esta experiencia se enmarca en la "III Convocatoria proyectos de innovación docente y premios a la Innovación" convocada por el Vicerrectorado de Ordenación Académica y Profesorado, a través del Centro de Formación Continua "Educa-Acción" pertenecientes a la Universidad Católica de Valencia, y se ha realizado a lo largo del primer semestre (de septiembre a diciembre de 2019). De ellos, 38 cursan la asignatura Habilidades Sociales y Dinámica de Grupos, del tercer curso del grado de Ciencias de la Actividad Física y del Deporte, y 48 cursan las asignaturas Fundamentos de la Educación Social y Tecnologías de la Información y la Comunicación, de segundo curso del grado de Educación Social. El rango de edad de los participantes es de 18 a 25 años, y con respecto al sexo, el 55,1 \% son mujeres y el 44,9\% son hombres.

\subsection{Instrumento}

Nos interesa conocer la percepción de los estudiantes sobre su aprendizaje en las competencias específicas de las asignaturas involucradas en el proyecto de ambos grados, acorde a sus respectivas memorias verificadas y plan de estudios. Por ello, el instrumento que se utilizó es la guía docente de cada una de las materias implicadas. De ellas se seleccionaron las competencias específicas y se solicitó a los estudiantes que valoraran cada una de estas con una escala Likert del 1 al 5 ( 1 = nada de acuerdo, $5=$ muy de acuerdo $)$. Ello nos permitió comprobar a través de la autopercepción del alumnado si la herramienta de gamificación contribuye a una mejor adquisición de dichas competencias en el proceso de enseñanza-aprendizaje del alumnado.

Las competencias específicas encuestadas en la asignatura de Fundamentos de la Educación Social son:

- Comprender e identificar los procesos históricos, socioculturales, políticos y legislativos que están en la consolidación de la profesión (PA_ FEDS1). 
- Conocer y analizar las políticas de bienestar social, los cambios sociales, económicos y la legislación que sustentan los procesos de intervención socioeducativa (PA_FEDS2).

- Analizar y diagnosticar las diferentes realidades complejas que fundamentan el desarrollo de los adecuados procesos socioeducativos (PA FEDS3).

- Mostrar una actitud empática, respetuosa, solidaria y de confianza hacia los sujetos e instituciones de educación social (PA_FEDS4).

Las competencias específicas encuestadas en la asignatura Tecnología para la Información y la Comunicación son:

- Adquirir los conocimientos y destrezas de algunos recursos informáticos y tecnológicos existentes en el ámbito socioeducativo (PA_TIC1).

- Ser capaces de diseñar recursos TIC propios (PA_TIC2).

- Promover actitudes positivas hacia el uso de las TIC en su ámbito profesional (PA_TIC3).

Las competencias específicas encuestadas en la asignatura Habilidades Sociales y Dinámica de Grupos son:

- Conocer y comprender los factores comportamentales y sociales que condicionan la práctica de la actividad física y el deporte (PA_HAB1).

- Conocer y comprender los efectos de la práctica del ejercicio físico sobre los aspectos psicológicos y sociales del ser humano (PA_HAB2).

- Aplicar los principios comportamentales y sociales a los diferentes campos de la actividad física y el deporte (PA_HAB3).

- Elaborar programas para la dirección de organizaciones, entidades e instalaciones deportivas (PA_HAB4).

Los análisis estadísticos se han realizado con el programa SPSS v23. Se han realizado estadísticos descriptivos para describir las características generales de la muestra y para obtener los promedios en función de las variables personales y contextuales contempladas. 


\subsection{Desarrollo de la experiencia}

Se ha diseñado la experiencia innovadora en forma de proyecto colaborativo interdisciplinar gamificado y se ha replicado paralelamente en las dos facultades de la Universidad. El proyecto se encuentra divido en tres fases de actuación bien delimitadas, con nivel creciente de dificultad y de implicaciónparticipación de los estudiantes con respecto a las tareas que hay que realizar. Los estudiantes trabajan por equipos de 4 o 5 componentes, permitiendo que se desarrollen ciertas competencias dentro del equipo que son importantes a lo largo de su vida profesional: dirigir proyectos, resumir temas, realizar presentaciones, etc., incorporando todas estas actividades a de su aprendizaje.

La narrativa a través de la que se construye el proyecto versa sobre una misteriosa "Organización Internacional Mundial (OIM)", que contactará de manera secreta con nuestra universidad y más concretamente con los grupos participantes en el proyecto. Esta OIM nos comunica que existe una amenaza real que pone en peligro la Tierra y que en unos años la realidad "física" desaparecerá y existirá solo una "realidad virtual digital". Mientras se toman acuerdos políticos y militares a nivel mundial, las universidades deben documentar digitalmente todas las secciones de vida humana y animal del planeta. La UCV ha sido seleccionada para, a través de sus estudiantes de Ciencias de la Actividad Física y del Deporte (o de Educación Social), realizar el proyecto de documentación digital de los colectivos de la sociedad española menos favorecidos o con problemas de integración social, racial, cultural o económicos. Este es el punto de partida de la experiencia innovadora realizada a partir de la cual se encomendarán tareas en forma de retos y misiones.

Los objetivos específicos planteados fueron los siguientes:

- Realizar un trabajo cooperativo y colaborativo mediante estrategias gamificadas.

- Utilizar las herramientas TIC para la investigación, desarrollo y creación de material digital propio.

- Investigar desde las competencias profesionales del educador social o del profesional de la actividad física y del deporte, en los diferentes colectivos sociales. 
Se ha desarrollado en las siguientes fases:

- Fase 1: Definición del colectivo utilizando herramientas digitales y creando un recurso con realidad aumentada.

- Fase 2: Investigación periodística sobre el colectivo. Trabajo escrito a modo de artículo sobre los datos e información recogidos.

- Fase 3: Creación de un vídeo documental con las etapas de preproducción (guiones técnicos, gráficos y literarios), producción o grabación y posproducción (efectos y montajes finales).

En cada una de estas fases se han establecido diferentes pruebas a modo de retos que los equipos debían completar para poder continuar con la siguiente fase. Se ha utilizado la plataforma Moodle como herramienta vehicular donde los estudiantes podían consultar en un tablero digital la fase en la que se encontraban, los puntos o recompensas obtenidas, pistas para la siguiente fase y resultado por etapas. Los materiales y recursos empleados durante este han sido variados y adaptados al entorno profesional del discente, utilizando mayoritariamente recursos TIC para fomentar y entrenar las competencias y habilidades digitales en los estudiantes. También se han puesto en práctica los elementos esenciales de la gamificación: roles de jugadores, retos, reconocimientos y por supuesto la narrativa conductora a lo largo del proyecto, fomentando desde el inicio la expectativa por saber qué va a ocurrir y despertando intencionadamente el interés del alumnado.

El procedimiento de recogida de la información, una vez finalizado el proyecto, fue mediante encuesta en papel contestada de manera anónima por los estudiantes.

\section{Resultados}

A continuación, se muestran el promedio de las percepciones de aprendizaje de los estudiantes en cada una de las once competencias específicas consultadas de las asignaturas involucradas en el proyecto.

Como se puede apreciar en la ilustración 1 , todos los indicadores que se miden sobre la percepción de aprendizaje del alumnado con respecto a las 
competencias específicas de las asignaturas superan la escala de 3. Los valores más altos (por encima del valor 4) hacen referencia a la competencia sobre "Mostrar una actitud empática, respetuosa, solidaria y de confianza hacia los sujetos e instituciones de educación social" $(4,53)$ y la competencia TIC sobre "Promover actitudes positivas hacia el uso de las TIC en su ámbito profesional" $(4,03)$. Muy cerca de estas valoraciones están "Conocer y analizar las políticas de bienestar social, los cambios sociales, económicos y la legislación que sustentan los procesos de intervención socioeducativa" $(3,93)$ y las otras dos competencias TIC, que son "Ser capaces de diseñar recursos TIC propios" $(3,88)$ y "Adquirir los conocimientos y destrezas de algunos recursos informáticos y tecnológicos existentes en el ámbito socioeducativo" $(3,86)$. La competencia de la asignatura Habilidades Sociales y Dinámica de Grupos mejor valorada ha sido "Conocer y comprender los factores comportamentales y sociales que condicionan la práctica de la actividad física y el deporte" con respecto a la asignatura del grado de Ciencias de la Actividad Física y del Deporte $(3,81)$, mientras que la menos valorada correspondiente también a esta asignatura fue "Elaborar programas para la dirección de organizaciones, entidades e instalaciones deportivas" $(3,54)$. El resto de las competencias se mantiene en un nivel similar (en torno a los valores 3,69 y 3,77 ).

En relación a la percepción de los estudiantes diferenciada por sexo (véase ilustración 2), a nivel descriptivo las mujeres obtienen mejores promedios que los hombres. A nivel cualitativo, encontramos que las mujeres tienen una mayor percepción del aprendizaje sobre todas las competencias específicas de las asignaturas implicadas en el proyecto, obteniéndose valores por encima de la escala de 4 en ocho de las once competencias evaluadas.

Cabe destacar en este caso que hay cuatro competencias en que las diferencias sobre la autopercepción del aprendizaje de las mujeres es 0,5 puntos de media por encima de la autopercepción de los hombres (PA_FEDS3, PA HAB1, PA_HAB2 y PA_HAB3), siendo la más diferenciada la de "Conocer y comprender los efectos de la práctica del ejercicio físico sobre los aspectos psicológicos y sociales del ser humano" (3,59 hombres y 4,25 mujeres) con una distancia de 0,66 (como puede observarse en la ilustración 2). 


\section{ILUSTRACIÓN 1}

Promedio de la percepción de los estudiantes respecto al aprendizaje de las competencias de las asignaturas

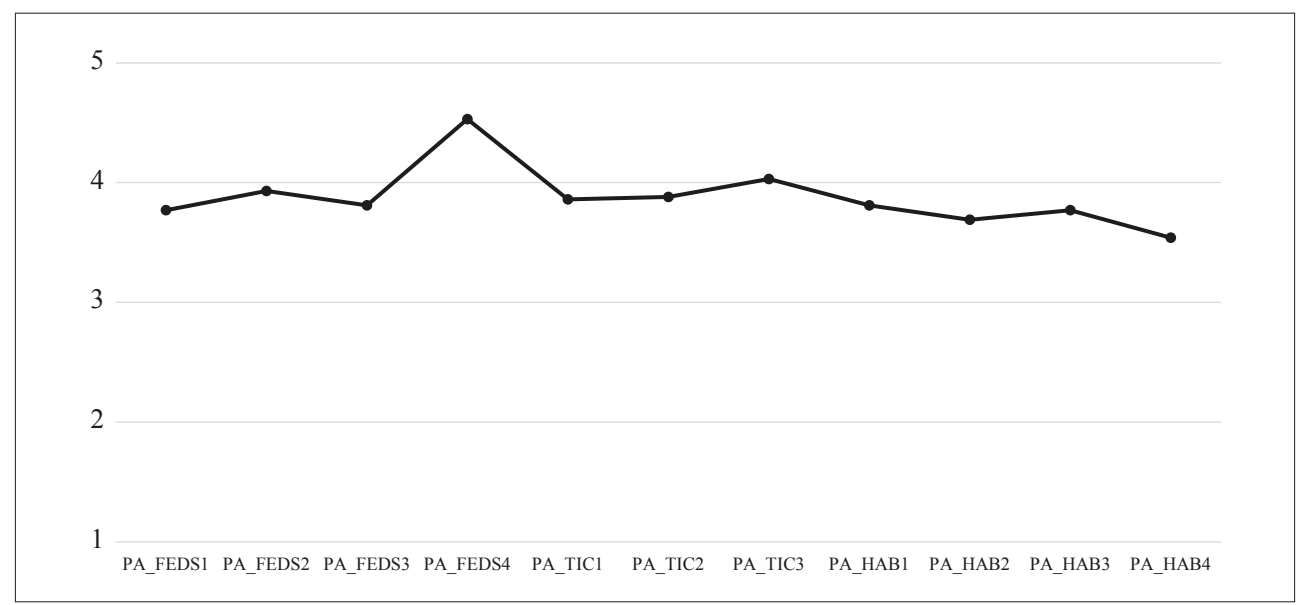

\section{ILUSTRACIÓN 2}

Promedio de la percepción de los estudiantes respecto al aprendizaje de las competencias de las asignaturas en función del sexo

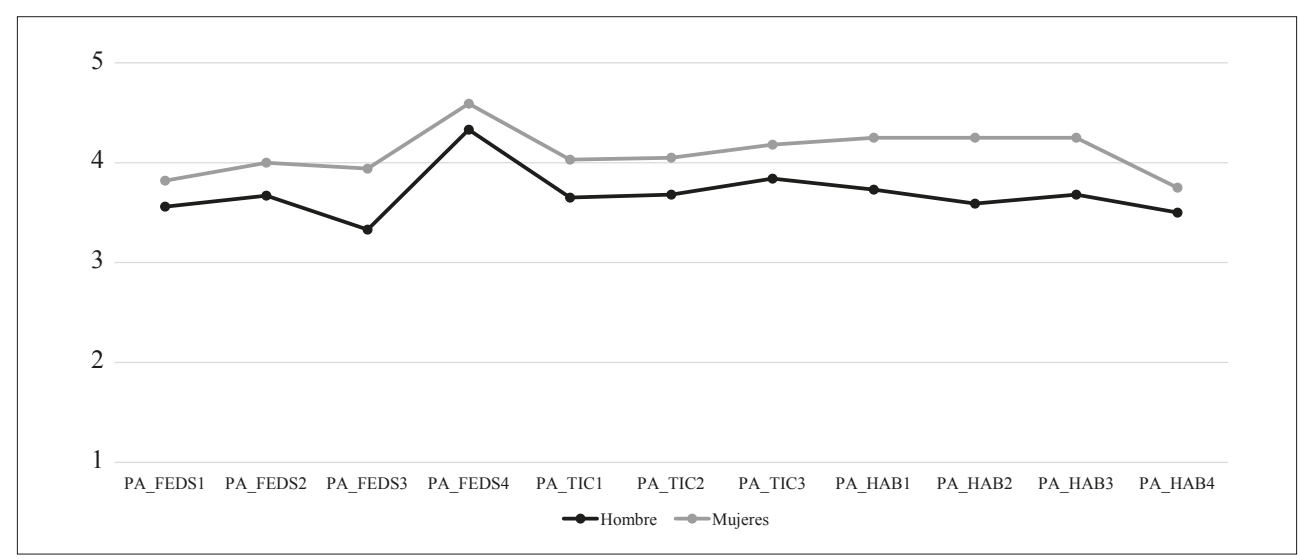




\section{DiSCUSIÓN Y CONCLUSIONES}

Esta propuesta de innovación docente se implementó con el objetivo de favorecer la adquisición de competencias específicas en el alumnado universitario de dos grados diferentes. Para ello, se puso en práctica la gamificación como metodología innovadora que propicia la implicación del alumnado en su proceso de enseñanza-aprendizaje con el fin de motivarles y hacerles partícipes de forma activa (Pisabarro y Vivaracho, 2018; Corchuelo-Rodríguez, 2018; Contreras y Eguía, 2016), así como el uso de las TIC para la investigación, desarrollo y creación de material digital propio.

Tras concluir el proyecto, se observa que con la aplicación de la gamificación, el alumnado percibe haber adquirido las competencias específicas descritas en cada una de las asignaturas implicadas en dicho proyecto interdisciplinar. Esta metodología didáctica tiene efectos positivos para el aprendizaje de conocimientos declarativos (saber qué), procedimentales (saber hacer) y actitudinales, coincidiendo con Zamora, Sánchez, Hipólito y Luque (2019), quienes demostraron que los estudiantes valoran muy positivamente el desarrollo de las competencias transversales, utilizando metodologías activas como la gamificación. Asimismo, Villalustre y Del Moral (2015) concluyeron en sus estudios que el $80 \%$ de los estudiantes declararon que con la ejecución del juego los estudiantes aprendieron conocimientos específicos de la materia, y adquirieron competencias tanto genéricas como específicas. Esto nos permite corroborar lo que ya han demostrado también autores como Carrión (2018), que afirma que el uso de la gamificación mejora la adquisición y desarrollo de las competencias propias del currículo.

Si nos detenemos en las dos competencias con mayor puntuación de nuestro estudio, podemos observar que la gamificación es una metodología adecuada para la adquisición de competencias actitudinales, como la empatía, el respeto hacia los demás o una actitud positiva hacia las TIC. Esto mismo han confirmado diferentes estudios llevados a cabo por Fernández, Porce, Nuviala, Pérez, Tamayo, Grao y González (2012); Robledo, Fidalgo, Arias y Álvarez (2015). Los resultados obtenidos por estos últimos autores mostraron que el 92,26\% de alumnado, valoraba positivamente el uso de las metodologías activas para el desarrollo de competencias relacionadas con las actitudes. 
Junto a estas competencias los datos estadísticos demuestran que las estrategias pedagógicas gamificadas utilizadas favorecen el diseño de recursos TIC y la capacidad para aplicarlas en el ámbito socioeducativo, tal y como también concluyó Guevara (2018). En el estudio llevado a cabo por esta autora, coincidiendo con los resultados obtenidos en nuestro estudio, se afirma que "los participantes lograron utilizar recursos para representar contenidos propios de la asignatura que imparten como mapas mentales, infografías, vídeos, comics, imágenes, evaluaciones interactivas y diseño de páginas web. También desarrollaron habilidades para editar contenidos ya existentes en la web y mejorarlos de acuerdo a las necesidades de sus estudiantes" (p. 54).

Otro aspecto que cabe destacar es la diferencia que existe entre hombres y mujeres, y son las mujeres las que autoperciben haber aprendido más en lo que respecta a competencias relacionadas con la materia. Los estudios de Prendes, García y Espinosa (2020) demostraron que las mujeres son especialmente más críticas en los aspectos metodológicos, y valoran más positivamente aspectos como los materiales preparados, los recursos utilizados y las expectativas generadas por el proyecto.

Con todo lo expuesto, se constata que el uso de las metodologías activas como la gamificación favorecen el desarrollo de competencias y capacidades en el alumnado, y son adecuadas para ajustar la formación académica a las necesidades sociales y profesionales actuales. No obstante, esta ha sido una experiencia concreta, con un grupo de alumnado reducido y esta circunstancia puede suponer una limitación en cuanto a extrapolar la experiencia. Pero, sin duda, supone también una oportunidad para continuar profundizando en el aprendizaje del alumnado universitario tal y como ya se ha iniciado.

\section{BiBLIOGRAFÍA}

BARnet, R. (2001). Los límites de la competencia. El conocimiento, la educación superior y la sociedad. Barcelona: Gedisa Editorial.

Bueno i Torrens, D. (2019). Neurociencia para educadores. Barcelona: Octaedro. 
Carrión Candel, E. (2018). El uso de la Gamificación y los recursos digitales en el aprendizaje de las Ciencias Sociales en la Educación Superior. Revista DIM 36.

Contreras, R. y Eguía, J. (eds.). (2016). Gamificación en las aulas universitarias (Bellaterra). Barcelona: Universidad Autónoma de Barcelona. Recuperado de: https://ddd.uab.cat/pub/1libres/2016/166455/Ebook_INCOM -UAB_10.pdf.

Corbo, J. C., Reinholz, D. L., Dancy, M. H., Deetz, S. y Finkelstein, N. (2016). Framework for transforming departmental culture to support educational innovation. Physical Review Physics Education Research 12(1). Recuperado de: https://doi.org/10.1103/physrevphyseducres.12.010113.

Corchuelo-Rodríguez, C. A. (2018). Gamificación en educación superior: experiencia innovadora para motivar estudiantes y dinamizar contenidos en el aula. EDUTEC, Revista Electrónica de Tecnología Educativa 63, 29-41.

De Miguel, F. M. (2006). Metodologías de enseñanza y aprendizaje para el desarrollo de competencias. Madrid: Alianza editorial.

Fernández, A., Porce, A., Nuviala, A., Pérez, R., Tamayo, J., Grao, A. y GonzÁlez, J. (2012). Estudio comparativo entre una metodología de aprendizaje tradicional respecto a una metodología de aprendizaje basada en el Learning by doing para la consecución de competencias específicas. UPO Innova: Revista de Innovación Docente 1, 1-11. Recuperado de: http://www.upo.es/revistas/index.php/upoinnova/article/view/95/90.

Fidalgo-Blanco, A., Sein-Echaluce, M. L., García-Peñalvo, F. J. y BalBín-Bastidas A. M. ${ }^{a}$ (2019). Método para diseñar buenas prácticas de innovación educativa docente: Percepción del profesorado. V Congreso Internacional sobre Aprendizaje, Innovación y Competitividad. Madrid.

García, C., Martín, M. L. y DíAz, E. (2019). Protocolo: gamificar una asignatura sin tecnología avanzada. Working Papers on Operations Management 10(2), 20-35.

González-Pérez, L. I., Ramírez-Montoya, M. S. y García-Peñalvo, F. J. (2019). Innovación educativa en estudios sobre el desarrollo y uso de la tecnología: Un mapeo sistemático, en M. S. Ramírez-Montoya y J. R. Valenzuela-González (eds.), Innovación educativa: Tendencias globales 
de investigación e implicaciones prácticas (pp. 171-195). Octaedro. Recuperado de: https://bit.ly/2Lzr0oa.

Guevara VizCaíno, C. (2018). Estrategias de gamificación aplicadas al desarrollo de competencias digitales docentes (tesis doctoral). Universidad Casa Grande (Guayaquil).

Leví-Orta, G. y Ramos-Méndez, E. (2013). Componentes de las competencias en los nuevos grados de algunas universidades españolas. Revista de Educación 362, 623-658. Recuperado de: http://www.educacionyfp.gob. es/dam/jcr:1 f886e15-27b7-44b3-9a5a-7bf7680a80a5/re36223-pdf.pdf.

Morin, E. (2001). Los siete saberes necesarios para la educación del futuro. Madrid: Paidós Studio.

Oliva, H. (2016). La gamificación como estrategia metodológica en el contexto educativo universitario. Realidad y Reflexión 44(0), 29-47.

Pisbarro Marrón, A. M. y Vivaracho Pascual, C. E. (2018). Gamificación en el aula: gincana de programación. Revista de investigación en docencia universitaria de la informática 11(1), 85-93. Recuperado de: http://www. aenui.net/ojs/index.php?journal=revision\&page $=$ article\&op $=$ download $\&$ path $\% 5 \mathrm{~B} \% 5 \mathrm{D}=402 \&$ path $\% 5 \mathrm{~B} \% 5 \mathrm{D}=593$.

Prendes-Espinosa, M. P., García-Tudela, P. A., Solano-Fernández, I. M. (2020). Igualdad de género y TIC en contextos educativos formales: Una revisión sistemática. Comunicar 63, V, XXVIII.

Ramírez-Montoya, M. y García-Peñalvo, F. J. (2018). Co-creación e innovación abierta: Revisión sistemática de literatura. Comunicar 54, 9-18. Recuperado de: https://doi.org/10.3916/C54-2018-01.

Ramírez-Montoya, M. y Lugo-Ocando, J. (2020). Systematic review of mixed methods in the framework of educational innovation. [Revisión sistemática de métodos mixtos en el marco de la innovación educativa]. Comunicar 65, 9-20. Recuperado de: https://doi.org/10.3916/C65-2020-01.

Real Decreto 1393/2007, de 29 de octubre, por el que se establece la ordenación de las enseñanzas universitarias oficiales. Recuperado de: https:// www.boe.es/buscar/pdf/2007/BOE-A-2007-18770-consolidado.pdf.

Rikkerink, M., Verbeeten, H., Simons, R. J., \& Ritzen, H. (2016). A new model of educational innovation: Exploring the nexus of organizational learning, distributed leadership, and digital technologies. Journal of Educational Change 17(2), 223-249. DOI: https://doi.org/10.1007/s10833-015-9253-5. 
Robinson, K. (2015). Escuelas creativas. Barcelona: Grijalbo

Robledo, P., Fidalgo, R., Arias, O. y Álvarez, L. M. (2015). Percepción de los estudiantes sobre el desarrollo de competencias a través de diferentes metodologías activas. Revista de Investigación Educativa 33(2), 369-383. DOI: http://dx.doi.org/10.6018/rie.33.2.201381.

Rogers, E. M. (2003). Diffusion of innovations. Free Press. Recuperado de: https://bit.ly/3dqBBO7.

Romero-LACAL, J, L. (2011). La educación en España: análisis, evolución y propuestas de mejora. Revista innovación y experiencias 42, 1-15. Recuperado de: https://archivos.csif.es/archivos/andalucia/ensenanza/revistas/ csicsif/revista/pdf/Numero_42/JOSE\%20LUIS\%20ROMERO\%20LACAL_1.pdf.

Villalustre, L. y Del Moral, M. (2015). Gamificación: Estrategia para optimizar el proceso de aprendizaje y la adquisición de competencias en contextos universitarios. Digital Education Review 0(27), 13-31.

Villa, A. y Poblete, M. (2010). Aprendizaje basado en competencias. Una propuesta para la evaluación de competencias genéricas. Bilbao: Universidad de Deusto.

Villa, A. y Poblete, M. (2011). Evaluación de competencias genéricas: Principios, oportunidades y limitaciones. Bordón 63(1), 147-170. Recuperado de: https://dialnet.unirioja.es/servlet/articulo?codigo=3601062.

Werbach, Kevin y Hunter, D. (2012). For the Win: How Game Thinking Can Revolutionize Your Business. Harrisburg: Wharton Digital Press.

Zamora-Polo, F., Sánchez-Martín, J., Hipólito-Ojalvo, F., Luque-Sendra, A. (2019). Utilización de la gamificación para el desarrollo de competencias transversales en el Grado de Ingeniería Mecánica, en Actas del 23rd International Congress on Project Management and Engineering (pp. 1957-1967). 